\title{
Capitalismo emocional: tensiones y solidaridades entre lo industrial y lo informacional
}

\author{
Elías Julián Molteni ${ }^{1}$
}

Recibido: 29/04/2021; Aceptado: 01/07/2021

Cómo citar: Molteni, E. J. (2021) Capitalismo emocional: tensiones y solidaridades entre lo industrial y lo informacional. Revista Hipertextos, 9 (16), 77-97. https://doi.org/10.24215/23143924e041

Resumen. En el presente trabajo analizaremos la incidencia de la racionalización, conmensuración y abstracción de las emociones en el entramado simbólico-prescriptivo del capitalismo. Para ello, proponemos establecer la continuidad y rupturas del denominado capitalismo emocional en el pasaje y reestructuración del industrialismo a la producción de bienes informacionales, es decir, desde el capitalismo industrial al informacional. En ese sentido, demostraremos cómo se fue configurando una serie de técnicas que dan coherencia a las emociones en la producción y reproducción del capital, particularmente cómo el dispositivo psicológico se inserta en la fábrica, mientras que otras tecnologías, específicamente ligadas al algoritmo y las nuevas disciplinas psi (psiquiatría biológica, psicología positiva, entre otras), tiene como espacio predilecto la empresa. A partir de ello, se diferenciará la conformación del sujeto: el individuo en el capitalismo industrial y el dividuo en el informacional. El objetivo de este trabajo es indagar en la emergencia del capitalismo emocional, en la etapa industrial, para explicitar algunos elementos epistemológicos, técnicos y simbólicos para su exacerbación y profundización en la etapa signada por lo informacional.

Palabras Clave: capitalismo emocional, disciplinas, emociones, gestión emocional, tecnología del yo.

Sumario: 1. Introducción. 2. Cartografiando las emociones y los capitalismos. 3. La familia llega a la fábrica y la fábrica a la familia: la psicología se difumina. 4. El poder embebido y encarnado: hacia el desplazamiento famacopornográfico del capitalismo. 5. Consideraciones finales.

\section{Emotional capitalism: tensions and solidarity between the industrial and the informational}

Abstract. In the present work we will analyze the incidence of rationalization, commensuration and abstraction of emotions in the symbolic-prescriptive framework of capitalism. For this, we propose to establish the continuity and ruptures of the so-called emotional capitalism in the passage and restructuring of industrialism to the production of informational goods, that is, from industrial to informational capitalism. In this sense, we will demonstrate how a series of techniques that give coherence to emotions in the production and reproduction of capital were configured, particularly how the psychological device is inserted in the factory, while other technologies, specifically linked to the algorithm and the new psi disciplines (biological psychiatry, positive psychology, among others), has the company as its favorite

\footnotetext{
${ }^{1}$ Licenciado en Sociología por la Facultad de Ciencias Sociales de la Universidad de Buenos Aires. Contacto: m95elias@gmail.com
} 
space. From this, the conformation of the subject will be differentiated: the individual in industrial capitalism and the dividual in the informational one. The objective of this work is to investigate the emergence of emotional capitalism, in the industrial stage, to make explicit some epistemological, technical and symbolic elements for its exacerbation and deepening in the stage marked by the informational.

Keywords: emotional capitalism, disciplines, emotions, emotional management, technology of the self.

\section{Capitalismo emocional: tensões e solidariedade entre o industrial e o informacional.}

Resumo. No presente trabalho iremos analisar a incidência da racionalização, comensuração e abstração das emoções no quadro simbólico-prescritivo do capitalismo. Para tanto, propomos estabelecer a continuidade e rupturas do chamado capitalismo emocional na passagem e reestruturação do industrialismo para a produção de bens informacionais, ou seja, do capitalismo industrial para o informacional. Nesse sentido, iremos demonstrar como se configuraram um conjunto de técnicas que dão coerência às emoções na produção e reprodução do capital, nomeadamente como o dispositivo psicológico se insere na fábrica, enquanto outras tecnologias, especificamente ligadas ao algoritmo e ao novo. as disciplinas psi (psiquiatria biológica, psicologia positiva, entre outras), têm a empresa como seu espaço preferido. A partir disso, será diferenciada a conformação do sujeito: o indivíduo no capitalismo industrial e o dividual no informacional. O objetivo deste trabalho é investigar a emergência do capitalismo emocional, na fase industrial, para explicitar alguns elementos epistemológicos, técnicos e simbólicos para seu agravamento e aprofundamento na fase marcada pelo informacional.

Palavras-chave: capitalismo emocional, disciplinas, emoções, gestão emocional, tecnologia do self.

\section{Introducción}

En este trabajo nos enfocaremos en la vinculación del capitalismo emocional y los procesos (re)productivos del capital, específicamente en las formas que se han desarrollado para la producción de una subjetividad ligada al trabajo en relaciones capitalistas, en las grandes etapas del capitalismo, industrial e informacional. Dejaremos de lado la etapa mercantil o preindustrial del capitalismo para enfocarnos en las sucedáneas dos etapas; nos centraremos en el transcurso del siglo XX, tomando como eje empírico más concreto la sociedad norteamericana, a raíz de los autores y las autoras que retomamos. Sin embargo, nuestro trabajo consiste en un análisis teórico crítico de las dimensiones normativas, prescriptivas, que enmarcan la relación del sujeto consigo, su cuerpo/emoción, en las relaciones capitalistas. Intentaremos leer en clave crítica la relación entre emociones, tecnologías del yo y procesos productivos capitalistas a fin de responder a las siguientes preguntas: ¿Cuáles son las construcciones del capitalismo emocional en el momento histórico del capitalismo industrial y del informacional? ¿Con qué medios tecnológicos se implican al yo en el proceso productivo capitalista en su etapa industrial y qué diferencia presenta en el capitalismo informacional? ¿Qué dimensiones - aspectos, relaciones, entornos - ponen de manifiesto las tecnologías del yo en cada etapa? ¿Qué tipo de saberes, técnicas y sujetos han dado conformidad la emocionalidad en el proceso productivo capitalista?

Para esto, decidimos hacer un análisis del poder racionalizador que se despliega en las etapas del capitalismo en referencia a las emociones, a cómo adquiere consistencia práctica (y teórica) en las emociones, la emocionalidad del yo y cómo se fue convirtiendo en un elemento más a tener 
en cuenta en la cadena productiva. Nuestra propuesta consiste en un análisis en la yuxtaposición de saberes y prácticas que conforman una racionalización de las emociones en el trabajo, una imagen del mundo y un tipo de estructuración subjetiva que son preponderantes en las etapas del capitalismo. El objetivo del trabajo consiste en determinar el cambio en la axiología del capitalismo que permita re-situar a las emociones dentro del entramado que propone dicha axiología; en definitiva, centrándonos en ella podremos advertir de forma más sistemática lo que atraviesa los cuerpos/emociones y, como tal, nos atraviesa. Como afirma Eva Illouz (2007, p.25), en la medida en que podamos centrarnos en procesos de constitución y regulación de las emociones - y los cuerpos, agregamos - podremos reconceptualizar el proceso capitalista de producción de mercancías, y viceversa. Para tal fin, hemos organizado el trabajo de la siguiente manera: en primer lugar, daremos un breve repaso a la conceptualización de las emociones y de los capitalismos a tratar, para luego, en segundo lugar, especificar las condiciones de emergencia del capitalismo emocional, en el industrialismo, tomando en consideración la ascensión de la psicología industrial, las técnicas de gestión y la propuesta como tecnología del yo. En tercer lugar, estableceremos las condiciones de emergencia de una nueva axiología, ligada a la atención, y las nuevas normatividades que dan conformidad al dividuo contemporáneo, correspondiente con una serie de saberes-técnicas que explicitaremos. Por último, retomaremos las conclusiones a las que hemos arribado. La hipótesis que se sigue es que el capitalismo emocional, oriundo del industrialismo, se encuentra exacerbado por las tecnologías digitales, por el influjo de tecnologías que constituyen lo que hoy denominamos capitalismo informacional. En ese sentido, lo dividual se esgrime como nueva lógica en que las acciones y los actores que, tendientes a la diferenciación, se encuentran, en términos axiológicos, forjados por el imperativo de la felicidad o la realización de sí mismo. A su vez, la exacerbación del capitalismo emocional condice con la transformación de los soportes de conocimiento que pasan de un indicio, un gesto, a un dato para retornar como sugerencia, como curso de acción posible-necesario, a través de traducciones que posibilitan el pasaje de una información digital a conductas, a un soporte de conocimiento de tipo subjetivo e intersubjetivo (Zukerfeld, 2010; 2017).

\section{Cartografiando las emociones y los capitalismos}

La sociología vio nacer a un objeto que pareciera quedar para otras disciplinas, las emociones. Ellas se perfilaron como objeto de estudio sociológico en lo se puede denominar como "giro afectivo", es decir la problematización de las emociones, afecciones y percepciones desde un entramado conceptual sociológico (Bericat Alastuey, 2000). Partiendo de los análisis de Sara Ahmed (2019), Eva Illouz (2007, 2008, 2009) y Adrián Scribano (2010, 2013) (si bien tienen puntos divergentes, no es posible referirlas aquí ya que exceden la propuesta de este trabajo) podemos conceptualizar a las emociones como un fenómeno relacionado a un cuerpo, y con ello, a una forma de entender y de estar en el mundo, de verse afectado por él y de conocerlo; las emociones tienen un carácter relacional y posibilitan, a la vez, relaciones con cosas y personas de forma concreta. Por otro lado, desde estas concepciones no son algo enteramente individual, sino que refieren siempre a una estructura o a procesos de normalización que configuran la relación del sujeto con su cuerpo y el modo en que se relaciona/actúa con el mundo.

Nos apoyamos en las lecturas de Scribano para pensar la "sociología de los cuerpos/emociones". En ese sentido podemos definir a los cuerpos/emociones como un 
continum y un resultado de una serie de articulaciones de diversos/plurales espacios/procesos que involucran determinaciones de lo concreto (Scribano, 2013, p. 94). El autor define al cuerpo como el locus de la conflictividad y el ordenamiento; en síntesis, a través y alrededor de él se da una serie de mecanismos de soportabilidad social correspondiente a las políticas de las sensibilidades, capaces de definir una geografía del cuerpo y una gramática de las acciones (Scribano, 2010). Entonces, las políticas de las sensibilidades perfilan afecciones, cognición e interacción, en la medida en que delimitan el mundo de lo sentible-observable-posible (Kogan, 2013). Scribano es tajante en esto y afirma que "Percepciones, sensaciones y emociones constituyen un trípode que permite entender dónde se fundan las sensibilidades. Los agentes sociales conocen el mundo a través de sus cuerpos" (2013, p.102). La geometría de nuestros cuerpos y la gramática de nuestras acciones, en tanto posibilidades, se fundan en este trípode atravesado, claro está, por procesos de estructuración social donde, entre ellos, cabe destacar las políticas de las sensibilidades y, particularmente en las actuales sociedades, la normalización a través del disfrute inmediato en el consumo. Tanto para Ahmed (2019) como para Illouz (2009) el cuerpo es una dimensión ligada enteramente a la emoción. Las emociones se encuentran vinculadas directamente con la estructura social de producción y cognición al reafirmar valores sociales e ideales de vida. La cuestión a observar es la producción y ligazón de esos cuerpos/emociones con los procesos productivos de los capitalismos.

\subsection{Los rostros del capitalismo}

Si, como propone Mariano Zukerfeld (2010; 2017), la explotación capitalista tiene como objeto la dimensión material/energética y cognitiva para con los trabajadores en las diferentes etapas del capitalismo, podríamos pensar la dimensión emocional como una tercera dimensión o, mejor dicho, como una dimensión entre lo energético/materia y lo cognitivo; entonces estas dimensiones son lo que el capitalismo explota de su fuerza de trabajo porque el capitalismo está de acuerdo con ello (Zukerfeld, 2010). En lo que respecta a las emociones, se observa en las mercancías y en la fuerza del trabajo cierta exigencia que configura lo que se denomina capitalismo emocional. Esta noción es conceptualizada por Illouz $(2007,2008)$ como una cultura, es decir, como un marco interpretativo de sí mismo que enlaza emocionalidad y economía. En definitiva, el capitalismo emocional engloba prácticas y discursos emocionales y económicos que se interrelacionan e intercambian (Illouz, 2007, p. 20). Esto se puede expresar en dos tipos de bienes; por un lado, los bienes culturales (reality show, talks show, libros de autoayuda, etc.) y, por otro lado, bienes que posibilitan una experiencia o un tipo de sensibilidad, "experiencias emocionales" (esto es en un sentido amplio, parten de un entramado que hará a cualquier bien un envase para una experiencia emocionante, más claramente puede verse en los bienes de consumo inmediato y el turismo) (Illouz, 2009). Desde otro punto de vista, el capitalismo emocional es una exigencia a la fuerza de trabajo para denotar emociones "positivas" (al menos desde la asunción de una tecnología del yo que se enfoca específicamente, antes que contener, en expresar y producir). Como se verá a lo largo de este artículo, la producción de trabajadores

\footnotetext{
${ }^{2}$ Hay que distinguir dos elementos de la positivización de las emociones: en primer lugar, la coherencia teórica y práctica que adquiere desde la década del '30, fundamentalmente en Estados Unidos, en los trabajos de Elton Mayo (retomaremos más adelante), es decir, el proceso de individualización del discurso sobre las emociones en las fábricas (Foucault, 2013). Por otro lado, desde la década de los '70 se empieza a distinguir "cabalmente" entre emociones positivas y negativas, teniendo un contenido moral, es decir, las emociones positivas son buenas, mientras que las negativas son malas (Ahmed, 2019).
} 
"felices" es algo que aparece embrionariamente en la década de 1930 para luego volverse un imperativo y un elemento para la conducción autodirigida del trabajador.

Pero, ¿a qué llamamos capitalismo industrial y qué diferencia se establece con el informacional? ¿Cómo se relaciona esa concepción del capitalismo emocional con estas dos etapas? En primer lugar, cabe destacar que la delimitación exhaustiva excede este trabajo. Podemos decir que el capitalismo industrial corresponde a un período histórico que engloba desde principios del siglo XVIII hasta el último tercio del XX, mientras que el capitalismo informacional se desarrolla desde la década de 1970 hasta nuestros días. El capitalismo industrial es asociado a las distintas maquinarias que tratan/trabajan niveles altos de materias y energía, mientras que el capitalismo informacional trabaja, esencialmente, con información digital, utilizada y enfocada en la producción de bienes informacionales (Zukerfeld, 2010). Sin embargo, esto no quita el carácter material de estos productos ya que el bit, unidad de información binaria y replicable que articula y equipara las informaciones, no deja de ser un ente con todas las letras, no deja de ser material (Cafassi, 1998), y no dejan de darse procesos de extracción de materias para su producción.

Si bien el concepto de capitalismo informacional puede ser matizado teniendo en cuenta los análisis que ponen de relieve el lado "cognitivo" de este modelo de acumulación, como los análisis de la escuela, justamente, del capitalismo cognitivo (Rullani, 2004; Moulier Boutang, 2011; Vercellone, 2013), sostenemos que, siguiendo a Zukerfeld (2010, 2017), este concepto deja de lado lo propio de esta etapa, que no es la preponderancia del conocimiento o de lo cognitivo, sino de la información y específicamente de la información digital. En ese sentido, el capitalismo informacional es la "Etapa del modo de producción capitalista signada por la contradicción entre relaciones sociales de producción orientadas a realizar a los tres tipos de bienes informacionales como mercancías, y el grado de desarrollo de las fuerzas productivas asociado a la ontología replicable de la información digital, que amenaza el status mercantil de esos bienes" (Zukerfeld, 2011b, p.3-4). Una de las cualidades indispensable de este tipo de producción es la replicabilidad de la información digital (ID) ya que refiere a la cualidad que tiene la ID de reproducirse con costos cercanos a cero. De allí que se esgriman diferentes estrategias de propertización del conocimiento (Zukerfeld, 2017) o que se comercialicen ciertos bienes informacionales como servicio, entre otras variantes (Srnicek, 2018). El insumo determinante es la ID, de ahí la producción de bienes informacionales $(\mathrm{BI})$, bienes obtenidos en procesos de producción con un peso relativo en la generación de o el acceso a la ID. Entonces, los BI tienen un alto nivel de conocimiento e información, mientras que los costos de producción en materia/energía son más bien despreciables. Tenemos así tres tipos BI; los primarios que están hecho enteramente por ID (tales como software, imágenes, sonidos, textos, etc.); los secundarios que presentan como rasgo distintivo la capacidad de almacenar, procesar y trasmitir ID (se puede establecer una distinción entre los que almacenan, como los chips y las computadoras, y los de trasmisión, como los semiconductores de silicio, etc.); y los terciarios que, por su parte, tienen como rasgo general que sólo la ID sea su insumo decisivo, consumen una gran cantidad de información, y corresponden a los productos de la industria farmacéutica, aplicaciones vegetales o animales de la genética, biotecnología, etc. (Zukerfeld, 2011b).

La organización de los lugares de trabajo ha cambiado a lo largo del siglo XX, pero en los últimos cuarenta años se experimentado una profusa y novedosa transformación. En ese sentido, la organización de la empresa ha pasado de una estructura predecible, jerárquica, rígida, 
arborescente y altamente burocrática, a ser un ámbito de constante cambio, flexible e impredecible (Cabanas, 2013); la nueva arquitectura de la empresa es reticular o en "red" (Castells, 2006). Así, la noción de carrera se ha vuelto obsoleta o, cuanto menos, problemática, porque su sentido como un camino ascendente y en línea recta ya no es el prototipo de las aspiraciones laborales, más bien, esto abre paso a una forma de "pasos de cangrejo", de ondulaciones horizontales (Sennett, 2000, p. 88). Estos cambios no sólo se pueden verificar en su acepción legal o formal, sino que se transformó en exigencias y prácticas de las empresas y de los trabajadores, afín a lo que Luc Bolstanski y Eve Chiapello (2002) llaman "nuevo espíritu del capitalismo", es decir, una nueva ética empresarial y laboral que se apoya fundamentalmente en la responsabilidad individual y en la psicologización de las personas. En ese sentido, Robert Castel ya había marcado el proceso de desafiliación como un proceso experimentado en las sociedades industrializadas avanzadas desde la década de 1970, como un proceso en que las "zonas" o espacios de seguridad de los sujetos se van corriendo al lugar de la incertidumbre generalizada (Castel, 1997, p. 13). Entonces, este aumento del riesgo o de la incertidumbre a nivel laboral introduce la inseguridad, la inestabilidad y la competitividad como una superficie donde el trabajador ha de demostrar lo que es (Cabanas, 2013, p. 256). Esto no es azaroso, dado el cambio de las tecnologías del yo ${ }^{3}$ que la empresa de finales del siglo XX introduce o, dicho de otra manera, dado que la empresa se vuelve alma, etérea (Deleuze, 1995).

Las diferencias en la organización también nos señalan el cambio en relación al reconocimiento. Según Bauman, mientras en el capitalismo industrial los dos polos eran el individuo y la sociedad, y el héroe era el que podía subsumir o destensar estos polos, en la etapa actual es la celebrity la que permite condensar las tensiones actuales (Bauman, 2009). Eso se debe a que, en el capitalismo informacional, dada la vorágine de información disponible, la atención es un bien escaso (Simon, 1971); la celebrity se configura como un tipo ideal porque más allá de estar conectado, el dividuo debe ser un nodo que capte la atención de otros dividuos (Zukerfeld, 2011a). Entonces, son estas series de cambios, especialmente la conectividad, el peso fundamental de las redes, de las conexiones y de los flujos de información/atención que circulan en ellos, los que permitieron dotar de una nueva forma al sujeto particular en el capitalismo informacional: el dividuo reemplaza al individuo del capitalismo industrial (Deleuze, 1995; Zukerfeld, 2010, 2011a).

Acaecida la década de los '70, el taylorismo y el fordismo pierden fuerza estructuradora de las relaciones de trabajo, un nuevo elemento emerge: el toyotismo o el modo de producción kan-ban. Los tiempos de producción se alteran, la demanda pasa a ser el elemento dinamizador en la producción, la innovación adquiere cada vez más relevancia en el mercado competitivo a escala global. Como se dijo, la forma reticular de la empresa reemplaza a la piramidal, y los estratos

\footnotetext{
3 Tomamos este concepto de Michel Foucault, quien afirma que las tecnologías del yo son las operaciones que permiten a los individuos, por ellos o por otros, efectuar una modificación en su cuerpo, en su alma, pensamientos, "o cualquier forma de ser" que le otorgue una transformación de sí mismo "con el fin de alcanzar cierto estado de felicidad, pureza, sabiduría o inmortalidad" (Foucault, 1988, p. 48). Así, a modo de apertura, podemos decir que las tecnologías del yo predominante en el capitalismo industrial serán, como lo demuestra Illouz, la psicología, en su variante industrial (Cabanas, 2013, p. 97-99) y el feminismo de la segunda ola (Illouz, 2007, 2008). Mientras que, rondado los años 1970, emergen o, al menos, se posicionan con otra fuerza las literaturas de autoayuda y, fundamentalmente, la psicología positiva (Illouz y Cabanas, 2019), a su vez, en el "comienzo de la psicofarmacología” (Foucault, 1988, p.144) por la década de 1950 tenemos otra variante como la psiquiatría biológica que, una vez introducidos los cambios tecnológicos "necesarios" para la visualización del cerebro, han establecido una concepción del yo neuroquímico (Rose, 2012) o, como intentaremos demostrar, un proceso de construcción de la subjetividad como motor del capitalismo farmacopornográfico (Preciado, 2008).
} 
medios son reestructurados: a la vez que hay desplazamientos, se elaboran nuevos métodos de implicación de los trabajadores inferiores con la empresa y se especializa la "carrera" de "recursos humanos". La teoría del capital humano, ligada a la gubernamentalidad neoliberal, irrumpe el escenario político, filosófico y económico desde fines de los años ' 60 . En ese sentido, cada trabajador es un empresario de sí mismo porque lo bumano es tomado como capital, es decir, como algo que produce su valor en la valorización de sí mismo (Foucault, 2004). En otras palabras, lo humano es reformulado como recurso, es decir, como algo maleable, explotable, descartable y valorizable. Se abre así la paradoja donde "los hombres son recursos y, por lo tanto, cosas, pero los recursos son humanos y, por lo tanto, no son simples cosas” (Marzano, 2011, p. 50).

Teniendo en cuenta este panorama general, el siguiente apartado tiene como fin explicitar las consideraciones del capitalismo emocional desde la década de 1930. A partir de allí, nos detendremos con mayor profundidad en la etapa informacional que aúna la producción de "experiencias emocionales".

\section{La familia llega a la fábrica y la fábrica a la familia: la psicología se difumina}

El capitalismo emocional tiene la cualidad de intercambiar o establecer relaciones entre las emociones y la economía; en todo caso, lo que permite esta cultura es la conmensurabilidad de las emociones. Este proceso se inicia en la proliferación de la narrativa terapéutica desde la década de 1920 que, junto con el feminismo de la segunda ola, permiten sacar de la intimidad las emociones y volverlas públicas: en tanto construidas desde marcos históricos y políticos más amplios y, por otro lado, como una puesta en publicitación de las emociones mismas. Las emociones son sacadas de su ámbito privado para demostrar su carácter de construcción pública y para denunciar las diversas violencias, particularmente enclaustradas en el cuerpo de la mujer (Illouz: 2007; Hochschild, 2008).

La narrativa terapéutica define un estilo emocional y una ontología de las emociones, es decir, establece la definición de la persona al darle marcos interpretativos para la vida en común, es parte de la narrativa de reconocimiento que produce identidad. La ontología emocional, entonces, será la jerarquización y articulación entre una variedad de emociones que se producen en la vida social del individuo, pero que, en cuanto individuo, parten de su singularidad y, este es el punto clave, son separadas del individuo, se exteriorizan (Illouz, 2007, p. 85). En este sentido, la singularidad de la persona para la narrativa terapéutica está enfocada en la familia. Esto es lo mismo que decir que el yo tiene un pasado o un punto de partida (cuestión sumamente importante para la diferenciación con otras tecnologías del yo). La teoría psicológica, posterior a Freud, inserta en la conceptualización del yo elementos que le otorgan un carácter maleable, permiten - en teoría - hacer un cambio sustancial en el yo ${ }^{4}$ (Cabanas, 2013; Illouz, 2007). Esa variación permite la conceptualización posterior sobre las herramientas para cambiar al yo, cómo ejercer sobre sí mismo una actividad para transformarse. En ese sentido, Edgar Cabanas señala:

\footnotetext{
${ }^{4} \mathrm{El}$ yo oscuro, misterioso, oculto dado por Freud comienza a flaquear en la mitad del siglo XX, surgiendo una serie de psicólogos que darán una visión del yo un tanto más maleable, donde se encuentra más localizable, teniendo un punto cúlmine, al menos para Illouz y Cabanas (2019), en lo que se denomina "psicología positiva". Aunque, como veremos, en la psicología industrial y, sobre todo, en la humanista, el yo oscurecido de Freud se difumina, adquiere un carácter maleable, modificable y, un término que se pondrá de moda a fines del siglo XX, plástico.
} 
Hay una individuación de la capacidad de transformación de sí, ya no será el mundo exterior que forje la identidad racional y el control del sujeto, sino que está en él los mecanismos para ello. Esta "autenticidad natural" comenzó a sustituir la idea victoriana de virtud, o sea el ejercicio de autocontrol aunado o fomentado con el ejercicio político público (Cabanas, 2013, p. 87).

\subsection{Hacia el paradigma de la comunicación}

La psicologización de la sociedad traslada el problema de vida privada, interna, a un problema de comunicación. El paradigma de la comunicación, sostenido por la psicología y por el feminismo, es rápidamente "captado" o introducido por la esfera económica. Muestra contundente de eso es el estudio de Elton Mayo (llevados a cabo entre 1924 y 1927 en una fábrica de General Electric) que se perfila como una herramienta para descubrir el verdadero "estado de ánimo" de la fábrica (Illouz, 2007; Sennett, 2000). En efecto, el análisis de Mayo sostiene que la fábrica gozará de una mayor armonía y productividad si se escucha o se da un ambiente con posibilidad de expresar sentimientos e inquietudes a los trabajadores. Mientras el gerente se presente como un agente de escucha, mientras más psicólogos se pongan en la fábrica, mayor será la capacidad productiva y habrá una disminución de tensiones y conflictos en ella; un mecanismo de soportabilidad social emerge en estas consideraciones (Scribano, 2013). Si en los albores del capitalismo industrial las instituciones de secuestro, ese vasto archipiélago disciplinario, constituyen una técnica de adiestramiento y disciplinamiento de sujetos para la producción de fuerza de trabajo (Foucault, 1980), el estudio de Mayo superpone dos tipos de instituciones: la médica-psicológica y la fábrica. La ortopedia social era el elemento necesario para mantener las relaciones productivas armonizadas $y$, en consecuencia, la actividad productiva funcionando plenamente; la explotación comienza a estar resguardada, entre otras regulaciones, por esta forma de tratar las emociones en el ámbito laboral. Los mecanismos de regulación en el capitalismo industrial de entre guerras suponen una introducción en las unidades productivas. Se lleva la psicología a la fábrica. El capitalismo industrial se propuso en este giro producir un registro emocional a fin de gestionarla y generar trabajadores más eficaces por medio de una asunción de cuadros medios tendientes a regular el trabajo en la fábrica, proceso señalado en la taylorización del trabajo (Szlechter \& Zangaro, 2020; Zangaro, 2011); sin embargo, puede decirse que la cuestión del registro de las emociones es parte indisoluble de la disciplina, del registro del detalle, de la graduación, del establecimiento de la norma y posterior normalización (Foucault, 2006).

En relación a lo que sostiene Illouz, sobre que el conflicto se verá modificado desde una concepción de la lucha por la escasez a la divergencia de las emociones en el ámbito laboral, Cabanas (2013) afirma:

Para los gerentes, la psicología industrial aportaba tanto nuevas formas de control del comportamiento de los trabajadores, mucho más sutiles, efectivas y con un carácter más marcadamente democrático, como la posibilidad de dirimir de forma técnica y dentro del espacio de lo psicológico - de los problemas familiares, de la personalidad y de la gestión de las propias emociones-, las deficiencias que generaba la propia estructura laboral. (2013, p. 102).

La "armonización" entre las condiciones democráticas experimentadas en la esfera pública y la reconceptualización de la vida íntima y laboral está enmarcada por el paradigma comunicacional. 
Este paradigma "exige" o predispone a los individuos a comunicar lo que sienten, produciendo una disolución de las características intrínseca de las emociones, esto es, ser indexicales y situacionales. Es decir, al volverse autorreflexiva y exteriorizada quita lo que hay de enérgico en la acción, quita el carácter emocional, más o menos indeterminado, de la acción; produce, en suma, una ontología emocional. Podemos decir que quita cierta fuerza a la emoción, pero más específicamente "implica que las emociones quedan cada vez más desvinculadas y desenlazadas de las relaciones y las acciones específicas y concretas. La condición para la 'comunicación' es, paradójicamente, la suspensión de los propios vínculos emocionales en una relación social" (Illouz, 2007, p. 90). La comunicación implica suspender o poner entre paréntesis la "cohesión emocional" en las relaciones. Entonces, en la fábrica, si bien contempla, da lugar a las emociones, las neutraliza a la hora de introducir el carácter necesariamente confeso de ellas.

En la ascendencia de las posiciones intermedias dentro de las fábricas, particularmente centrada en la posición de los psicólogos, el taylorismo impulsa un control de la emocionalidad de los empleados y también una racionalidad de los esfuerzos a través del cálculo y de la ciencia. Esta "influencia de los estados de ánimo" por medio de la organización científica del trabajo tiene un punto de anclaje en la expansión de puestos medios por parte de gerentes, psicólogos e incipientes managers (Zangaro, 2011). Entonces, si el taylorismo permite una racionalización del tiempo y de las energías (físicas y emocionales) en el trabajo, el fordismo es su instrumento de imposición. A través de la utilización intensiva de maquinas, la racionalización del trabajo, y la integración vertical de la producción, el fordismo estableció elementos para mermar la conflictividad laboral, el ausentismo y, en definitiva, fomentar la productividad de la empresa. Para estos objetivos, la unión fordismo-taylorismo implicó la expansión y el fomento de la "adhesión a la cultura de la empresa", con un fuerte carácter moralizador y, por otro lado, estableció una suerte de pacto interclasista en donde la elevación de los salarios y la seguridad social eran claves para la constitución del periodo de acumulación welfarista (Zangaro, 2011, p. 165). Sin embargo, será durante la década del '60 y '70 donde el discurso managerial adquirirá fuerza.

Como señalamos anteriormente, los trabajos de Elton Mayo fueron un antecedente en la ascendencia y/o superposición entre la empresa y la nueva cultura terapéutica. En las décadas siguientes continúan las investigaciones. A través del enfrentamiento y el control de la incertidumbre, la racionalización que proponen estas perspectivas reside en la producción de técnicas que puedan evaluar el desempeño y la productividad, las motivaciones y las afecciones de sus empleados. En resumen, estas propuestas se basan en la "hipótesis de que un trabajador feliz, con buen equilibrio personal, contribuye a crear una buena moral de grupo, y esa buena moral contribuye de manera positiva en el incremento de la productividad" (Zangaro, 2011, p.168).

Ahora bien, en el capitalismo industrial la noción de carrera laboral "apaciguaba" los efectos del sacrificio. Además, aunque la libertad individual comenzará a ser definida de forma positiva, la narrativa terapéutica producía una reubicación del yo en relación a la familia. Otorgando un carácter en contexto o, en otras palabras, el carácter como un efecto de las relaciones familiares, las tecnologías del yo en el industrialismo situaban al yo en un entramado más amplio, con jerarquías y con ciertos valores que están más allá del individuo- de ahí que el tipo ideal del capitalismo industrial sea el béroe, aquel que puede condensar la totalidad en sí o, en todo caso, el individuo que refiere a una totalidad (Bauman, 2009). Esta concepción parte de la idea 
"republicana" de la libertad del yo que, para los liberales, implicaba siempre una totalidad política-cívica (Cabanas, 2013).

La creciente influencia de la psicología y del feminismo coloca al yo en una posición de extrema publicidad, lo coloca en lo público en tanto es producto de múltiples relaciones y discursos. A partir de la década de 1940 los films y la literatura van estableciendo paralelismos entre una vida individual plagada de emociones y el intento creciente de controlarla. La popularización de la psicología, y la consecuente psicologización de la sociedad, encuentra en estos productos culturales sus medios más idóneos para su puesta en práctica. A través de una serie de procedimientos se genera la racionalización del valor de la personalidad. Racionalización que no está relacionada con un saber hacer específico del trabajador, sino que son "metahabilidades" que determinarán las posibilidades de insertarse "adecuadamente" en el proceso de producción como fuerza de trabajo, y que conllevan posibles relaciones interpersonales desde un apaciguamiento de las emociones.

Esto nos lleva a uno de los efectos centrales para Illouz - y en cierto sentido para Ahmed sobre la puesta en prácticas de estas técnicas. En primer lugar, Illouz $(2007,2008)$ sostiene que la narrativa terapéutica produce aquello que quiere erradicar: al centrar su atención en los dolores que ha transitado el yo, la narrativa terapéutica produce el dolor que pretende desterrar. Por otro lado, el discurso terapéutico coloca a las emociones en condiciones externas al sujeto, como dijimos quita su carácter inmediato e indexical, produciendo así una paradoja: mientras coloca un imperativo de gestionar su expresión a través del paradigma comunicacional, pone afuera del sujeto las emociones, las hace externas (Illouz, 2007, p. 81). En tercer lugar, los valores presentados, para Ahmed (2019) la felicidad se constituye como promesa, como un espectro de la falta y la posibilidad de colmarla. La división interno-externo es parte de la concepción de la ortopedia social que opera en el industrialismo (Preciado, 2008).

Ahora bien, la división público-privado comienza a ser problematizada. Hochschild sostiene que la "gestión de las emociones" deja su ámbito privado para ser un acto público, una puesta en valor y en circulación donde es comprado en un lado y vendido en otro. Por ende, tenemos que la gestión no es un intento espontáneo de modificación (emotion work) sino un "plan predeterminado y coactivo (emotion labor)" (podemos ver la cercanía con el planteo de Illouz) (Bericat Alastuey, 2000, p. 162). Por otro lado, las normas emocionales dejan la discrecionalidad de la negociación privada para estar formalmente publicadas (en diferentes soportes objetivos, podemos decir) en manuales, en guías profesionales, en películas y demás productos culturales. Por lo tanto, el trabajo emocional público se posiciona sobre el privado y, consecuentemente, "El trabajador debe controlar sus emociones, pero las compañías le piden, además, no sólo una actuación superficial, sino una profunda, no sólo que sonrían, sino que sientan la amabilidad que ofrecen y que disfruten con la sonrisa" (Bericat Alastuey, 2000, p.163).

Podemos concluir el apartado señalando la emergencia del capitalismo emocional en uno de sus puntos nodales: la psicología industrial y el feminismo de segunda ola, ambos basados en el paradigma comunicacional. La construcción de un sí mismo y de las capas medias con capacidad para encausar la buena gestión de las emociones, es un hecho que se irá problematizando en las décadas siguientes por medio de la crisis de la fábrica y la emergencia de un nuevo discurso empresarial. En los albores del capitalismo emocional, la inteligencia cognitiva cede lugar ante una ontología de las emociones que prescribe su buena gestión para adecuarse al contexto. Luego, como veremos en el siguiente apartado, la empatía, el tacto, la escucha o la inteligencia 
emocional, se volverán una precondición del trabajador hasta marcar a fuego las percepciones de sí mismo. En ese sentido, la producción del capitalismo contemporáneo no está volcada enteramente a la producción de mercancías, de bienes consumibles, sino, más bien, tiende a la producción de la subjetividad y explota (además de la fuerza física e intelectual) la fuerza de correrse, la potentia gaudadi ${ }^{5}$ (Preciado, 2008), cuestión a analizar en el siguiente apartado.

\section{El poder embebido y encarnado: hacia el desplazamiento famacopornográfico del capitalismo}

Con la sanción de la ley de salud mental establecida en 1946 por parte del gobierno de Estados Unidos, se plantea el problema de la emocionalidad según la mirada médica-psicológica donde las patologías psíquicas condensan una incapacidad práctica de respuesta ante determinada situación. En otras palabras, las emociones en esta ley estarán reguladas como conductas establecidas en determinadas situaciones; en todo caso será una antesala de la expansión médica elaborada por el Manual diagnóstico y estadístico de los trastornos mentales (DSM, por sus siglas en inglés). En esta ley se amplía el campo de trabajo de las narrativas terapéuticas, reclamando como objeto de estudio y como campo de intervención la salud mental de todos los ciudadanos. A raíz de la positivación de la psicología postfreudana surge la noción de "inteligencia emocional", una capacidad del individuo para atenuar o exacerbar las emociones según el contexto. Por lo tanto, una de las fuertes tendencias desde estos momentos, y aún más con el auge de la psiquiatría biológica y de la neurociencia es la capacidad del individuo de aislarse del entorno por medio de estas habilidades o suscitaciones de estados mentales/afectivos.

Entre los años 1860 y 1905 una teoría sobre la comunicación corporal tomó fuerza y permitió que algunas nociones vayan adquiriendo estatuto de objeto, en particular la "hormona". A partir de ello la teoría hormonal dará impulso a tratar al cuerpo como un sistema de comunicación. El cuerpo será, entonces, "el efecto material de estos intercambios semiotécnicos" en tanto que las hormonas son informaciones, códigos vivos que permiten actuar en otras partes del cuerpo, actuar a distancia (Preciado, 2008, p. 122). A partir de estos años comienza, y crece increíblemente, la comercialización de materiales orgánicos. El tráfico de fluidos humanos se da entre diferentes instituciones disciplinarias (cárceles, hospitales, mataderos) que terminan formando parte del sistema de producción de cuerpo-capital; estas instituciones, claro está, obtienen réditos económicos por estos flujos vivos. A mitad del siglo XX se dan dos inventos, la pastilla antibaby en 1951 y el Viagra unos años después, que tendrán un viraje clave en esta resumida historia de capitalización del cuerpo: los dos inventos sacan de la intimidad, de lo

\footnotetext{
${ }^{5} \mathrm{Al}$ decir de Paul. B. Preciado lo que el capitalismo actual pone a trabajar es la fuerza de correrse. Esta fuerza es la propulsora de un placer-con. Es algo que aspira a extenderse en el tiempo y en el espacio, a todos y a todo, y que no puede encapsularse, privatizarse en el sentido de adquirirlo como propiedad, sino que se pierde en cada experiencia; consumo y producción de la fuerza de correrse se efectúan en el acto. Esta comercialización de la potentia gaudandi no puede darse sin el control técnico de la reproducción de la especie y de su reproducción en tanto fuerza susceptible de ser capitalizada (Preciado, 2008).

6 Si bien el concepto "inteligencia emocional" es popularizado por Daniel Goleman en la década de 1990, para Illouz (2007, p. 142) este concepto cierra, o condensa, lo que ya se veía desarrollando desde la primera post guerra, es decir, un proceso de redefinición de la interioridad humana que desligase de la centralidad a la razón y a lo consciente, produciendo conceptos y técnicas que permitan la elaboración y la gestión de la competencia emocional; este concepto permite clasificar a las personas, pero sobre todo permite quitar la carga energética, cosa ya experimentada por el paradigma comunicacional, produciendo una división interna en el propio individuo o, mejor dicho, dividiendo a la persona según las emociones y la competencia en la situación que se encuentre.
} 
privado, la gestación y la "masculinidad" para volverse una técnica/producto capaz de ser conseguidos en una farmacia, pasan a estar en "un dominio de capitalización y de ingeniería política" (Preciado, 2008, p. 126). En ese sentido, Preciado afirma que el capitalismo actual "no produce cosas", sino que, en cambio, de lo que se trata es de inventar subjetividad y de producir "ideas móviles, órganos vivos, símbolos, deseos y reacciones químicas y estados del alma" (Preciado, 2008, p. 47). Pero no es hasta la década de los setenta y ochenta que se desligará el tratamiento psicológico de las emociones a uno farmacológico, desplazando, en cierta medida, la disciplina psicológica por la psiquiatría biológica. El ejemplo paradigmático es la fuerza clasificatoria y el afán de definición que tiene el tercer DSM (1980) donde se permite replantear la cuestión de las emociones a una cuestión fisiológica, específicamente, a una disfuncionalidad de elemento electroquímico (Rose, 2012).

Pasamos de una disciplina de los cuerpos en tanto tecnologías ortopédicas a que el poder se exprese a través de los cuerpos, de sus flujos deseantes y su excitación. Teníamos, en la idea de que cada cuerpo es uno a corregir, sistemas "duros y externos de producción de subjetividad. Se trata[ba] de ortopedias políticas, de exoesqueletos disciplinarios" (Preciado, 2008, p. 63). En la era farmacopornográfica, en cambio, pasamos a un cuerpo prótesis, una dimensión políticotécnica anclada y expresada en el cuerpo (sensu Haraway); las tecnologías se hacen cuerpo, se incorporan como parte íntegra de él ${ }^{7}$. Entonces, en esta sociedad "el modelo de acción sobre el cuerpo es la microprostética: el poder actúa a través de una molécula que viene a formar parte de nuestro sistema inmunitario" (Preciado, 2008, p. 67) y demás elementos "naturalizados" en nuestros cuerpos. El cuerpo ya no habita lugares disciplinarios, sino que es habitado por ellos.

Sin embargo, a la par que esta medicalización de la vida psíquica, del desarrollo tecnológico que pone a disposición del yo una conceptualización neuroquimica de sí mismo, de esta incorporación de los mecanismos de normalización que otrora quedaba en manos de un agente externo, surge otra corriente de la psicología que pondrá de manifiesto otro concepto: ya no el dolor, sino la felicidad (Illouz \& Cabanas, 2019). La felicidad ha adquirido una dimensionalidad central para la política de las sensibilidades, todos los cuerpos/emociones, se dice desde diferentes ámbitos o instituciones, deben tender a ser lo más felices posible. La felicidad se ha vuelto el criterio para la autopercepción en y ante el mundo, es el elemento para definir una "vida bien vivida", una vida aprovechada, y se ha configurado como la promesa más "factible" y la más deseable (Ahmed, 2019). La ciencia de la felicidad y sus diferentes profesionales parecen seducidos por una tendencia a conmensurar un estado de ánimo con un tipo de cálculo, con técnicas y con discursos que exaltan la capacidad adaptativa de la persona. Por el momento, una parte importante reduce la posibilidad de ser felices en la capacidad para afrontar los embates de la vida y de aprovechar las oportunidades que se nos presentan (Ahmed, 2019). Por eso, la narrativa de la felicidad adquiere un sentido de lucha. Esta lucha se da en un sentido retrospectivo (donde lo "negativo" queda atrás, estableciendo como valor central la "resiliencia") y otro sentido proyectivo (un ejercicio constante en sí mismo para cambiar en post de ser mejores) (Illouz \& Cabanas, 2019). Por eso, la apreciación de Dardot y Laval (2013, p. 338) se vuelve valiosa; ellos afirman que en la nueva empresa el trabajador tiene un carácter guerrero, tiene que competir consigo mismo (con su pasado y con sus valoraciones) y con los demás en el mercado laboral.

\footnotetext{
${ }^{7}$ Este es un sentido similar al propuesto por Sibilia, para quien el desarrollo del capitalismo en la presente etapa no solo está embiode (encarnado) en un cuerpo determinado, sino que está embiedde (embebido) en una cultura intersubjetiva (Sibilia, 2008, p. 20). Aunque, cabe aclarar, para la autora este aspecto no está presente únicamente en la actual etapa del capitalismo.
} 
$\mathrm{Al}$ igual que el guerrero deseoso de mayor honor y víctimas en su haber, el empresario de sí volcado a la felicidad siempre puede (debe) ser más feliz, la presencia de bienestar debe ser continua y siempre encaminarse a más. En ese sentido, al igual que la psicología del capitalismo industrial, la ciencia de la felicidad (y su promesa-imperativo constante) produce aquello que pretende resolver, genera malestar continuo en el momento que pretende generar bienestar o felicidad. Entonces, al producir un discurso que tiende a la autorrealización se produce una narrativa de la enfermedad, es decir, que siempre habrá algo que cambiar, que combatir, que producir para ser mejor. La narrativa terapéutica se apoya en una narrativa de la enfermedad, más aún cuando define un ideal de forma ambigua, indeterminado y que no finaliza en un punto concreto (Illouz, 2008; Illouz \& Cabanas, 2019; Ahmed, 2019).

A diferencia de la incipiente psicologización que el rol de los psicólogos sostenía, en el mundo de la empresa actual está en cada cual establecer la gestión de las emociones y proceder en los cambios para su mejora. Esto es el efecto de, al menos, tres variaciones en la narrativa terapéutica: por un lado, la definición de la normalidad no es la ausencia de dolencia o de patología, sino la perseverancia en ser la mejor "versión" de sí mismo, el individuo que no quiere cambiar será patologizado, lo que se le pide es que no muestre incompetencia para poder modificar su presente. Por otro lado, la noción de trauma, que establece un recorrido del sujeto, establece la historia por la cual el sujeto "devino" enfermo, es reemplazado por la asunción de que no hay un punto de partida, de que se vive en un presente permanente, lo que demuestra el carácter disfuncional de la persona es su incapacidad de modificar su presente, de proyectar un futuro mejor o, en todo caso, no poder superar su pasado. Por último, mientras el trauma, y la enfermedad consecuente, tenía un punto de resolución en la identificación del problema y, en caso más extremo, la cura, en la nueva narrativa terapéutica no hay punto de llegada, porque la felicidad, el gran e indefinido objetivo, no culmina, no tiene punto de culminación. Por lo tanto, el sujeto del capitalismo actual produce una ultra-subjetivación, esto es, la necesidad de transcenderse constantemente a sí mismo, donde no hay punto o estado último y estable de "posesión de sí mismo”, sino un ir constantemente más allá de sí (Dardot \& Laval, 2013, p. 362).

\subsection{E1 dividuo y el mercado de trabajo}

La constante reformulación de sí mismo, accionar indispensable para el motor del capitalismo, está en íntima relación a las exigencias que contempla Sennett en el mundo laboral, como la capacidad de desprenderse del pasado, de aceptar de forma necesaria la fragmentación y de fomentar o fomentarse la espontaneidad; son estos los tres elementos del "sistema de poder flexible que corroe el carácter" de los empleados, sea de la índole que sea (Sennett, 2000, p. 65). Siguiendo con este autor, se puede decir que este sistema de poder flexible hace que el riesgo se vuelva "normal y corriente" (Sennett, 2000, p. 85). Son esas "habilidades blandas" (Zangaro, 2011) o "metahabilidades" (Zukerfeld, 2017) las que determinan el valor de tal o cual trabajador que, en definitiva, tiene que ser "flexible", es decir, estirarse sin romperse. No es muy diferente, sacando a un costado los puntos de partida y las implicancias teóricas que se producen de ello, con la idea de modulación en Deleuze. En efecto, este proceso incesante, inacabado, es un "cambio autodeformante" que implica la transformación del sujeto en otro tipo: el dividuo (Deleuze, 1995, p. 249). Esas metahabilidades no pueden realizarse si no hay un corte, un momento de diferenciación con un sí mismo. El imperativo del feedback y de la asistencia de algunas tecnologías del yo (como pueden ser el coaching, la psicología positiva, la psiquiatría biológica) que subdividen 
al sujeto, lo fragmentan e intentan reconstruirlo bajo una soberanía específica: la realización de sí mismo por medio del lenguaje (coaching), de la búsqueda de la felicidad (psicología positiva) o una parte de sí devenido en sustancia, compuesto, que se intervendrá para restablecer el conjunto (psiquiatría positiva) (Marzano, 2011; Illouz \& Cabanas, 2019; Rose, 2012).

"Los individuos han devenidos 'dividuales", afirma Deleuze (1995, p. 251) y esto específicamente por el peso que se otorgan a, por un lado, la conexión y, por otro, la atención. Por ello, se erige como condición la conectividad (sin ella no habría conexión posible y menos flujos de atención a la información que se entrega-produce). Así como el capitalismo industrial hace flaquear aquello que consume sus bienes prototípicos, el caso más concreto son las reservas de petróleo en una "sociedad del automóvil", en el capitalismo informacional, los flujos de información digital por doquier y a cualquier hora, vuelven escasos los flujos de atención, aquello de que se alimentan (Simon, 1971; Zukerfeld, 2011a). El exceso de información produce la escasez de atención y la necesidad de gestionarla eficientemente entre las fuentes de información abundante que existe; de ahí que Google, una empresa insignia del nuevo capitalismo, sea un gran gestor de la atención. De forma sencilla, se puede afirmar que "en el mundo de Internet el poder axiológico de las conexiones y la atención se aprecia de manera prístina. Estar conectado y concitar interés son bienes sumamente valiosos" (Zukerfeld, 2011a, p. 3). El paroxismo de esta escasez es la consecuencia lógica del sintagma $24 / 7$, en donde no hay vacío, donde se combina la exigencia de brindar información y la red de observación que todo lo ve, o que al menos lo intenta (Crary, 2015). Un ejemplo claro, sin duda no el único, es el de JenniCam, hecho en 1997, que había dispuesto en su departamento un conjunto de cámaras que enviaban videos las 24 horas del día, los siete días de la semana, a su página web (Sibila, 2008, p. 242) ${ }^{8}$.

Justamente, el proceso de generación de bienes informacionales primarios por parte de una nueva categoría que puebla gran parte de las redes digitales, los prosumidores ${ }^{9}$, vuelve a la atención un bien escaso, y es así necesariamente porque de ahí el valor de un bien en vez de otro: el que recolecte o "estimule" más interacciones y más conexiones será el bien más valorado. Sibilia (2008) desarrolla una etnografía sobre los y las bloggers para retratar el valor de la atención del otro. Sin embargo, no es solo valiosa la atención del otro sino que lo "propio", la intimidad, adquiere valor, se valoriza y mercantiliza, en la publicitación. Ahora bien, el paradigma comunicacional sostenido desde el primer cuarto del siglo XX es redefinido en los albores de internet y del capitalismo informacional. En ese sentido, en la multiplicidad y profundidad de las redes existen diversas formas de presentarse ante el otro abstracto que supone lo digital. Sin embargo, lo que se esgrime como condición es el imperativo de "muéstrate a ti mismo", es decir, la prescripción de demostrar ante otros lo que tiene de singular el usuario-sujeto (Remondino, 2012). A su vez, esta expresión, sostenemos, no puede entenderse sin el "transciéndete a ti

\footnotetext{
${ }^{8}$ Es importante resaltar lo que señala Sibila, en ese momento Jennifer Ringley, la joven que exhibía su intimidad mediante las cámaras señalaba que le gustaba "sentirse observada". Pero, justamente esto es algo a resaltar, en ese momento la exposición de la intimidad se veía como algo extravagante que tenía que ser justificado. Como señala Crary, esa exposición que el concepto $24 / 7$ engloba, no corresponde sólo a los días de la semana sino, sobre todo, "la extinción de las tinieblas y la oscuridad" (2015, p. 60).

${ }^{9}$ Este concepto se relaciona con el modo de producción de bienes informacionales centrado en la disminución de los costos de los mismos; es decir, es parte de los mercados que entregan sus productos gratuitamente mientras obtengan sus ganancias vía publicidad. Un ejemplo paradigmático, no el único, es la plataforma de contenidos Youtube, donde una serie de trabajadores que producen y "suben" sus videos son a la vez consumidores de la red social. A su vez, "prosumidores" refiere a aquellos que tienen los "medios de producción" para, justamente, producir aquello que se consume: una cámara con micrófono y una conexión "decente" puede ser suficiente para que uno ingrese al mundo de "hacedores de contenido" (Zukerfeld, 2011b).
} 
mismo" como una operación que invita/exige la constante reformulación de aquello que se puede ofrecer en las redes, de aquello dirigido para otros. Como adelantamos, esto es demostrado por Sibilia en cuanto que la interioridad está atravesada por una lógica de la publicación o, más específicamente, de la ex-timidad, es decir, la exhibición de lo interno o lo íntimo en la red, transformándolo en un flujo de información digital capaz de ser mercantilizado (Sibilia, 2008).

Entonces, estas corrientes nos sugieren que la subjetividad se encuentra, en relación a la nueva posición de las tecnologías del yo, en una disposición numérica, esto es, que son ante todo una configuración de cantidades. Esas cantidades, claro está, se trasmutan en cualidades: estar feliz, ser mejor, crecer o florecer, etc.; por ello, la consecuencia lógica señalada por Deleuze de devenir dividuales, tanto para las ciencias del cerebro como para nuestra cotidianidad en la red y para las tecnologías psicológicas actuales (particularmente la psicología positiva), estamos realizados de pequeñas cantidades que se trasmutan en cualidades, cualidades emocionales. Si seguimos esta conclusión, podemos observar que la conmensurabilidad del capitalismo emocional se encuentra aún más arraigado, prescribiendo acciones y estableciendo relaciones.

La exaltación del paradigma comunicacional se encuentra de la mano de una "tecnoideología", en palabras de Sadin (2020), que produce una homologación entre procesos cerebrales, afectivos y emocionales, y las lógicas económicas y sociales. En definitiva, es el proceso de traducción (Zukerfeld, 2017) el que permite la relación estrecha entre características personales, deseos, necesidades coyunturales, etc. y una producción de información, de conocimiento codificado. A través de esta operación, en un segundo momento (por lo demás, instantáneo) se produce una sugerencia, un cierto empuje a tal o cual curso de acción; estamos ante el poder iniciático o conminatorio de las tecnologías digitales (Sadin, 2020). Si bien el capitalismo informacional exalta ciertas tendencias surgidas bajo el amparo de la fábrica industrial, constituye puntos de ruptura con respecto a la etapa de acumulación precedente, principalmente en lo referido a la posibilidad de reproducción, a la tercerización de los bienes que pone en juego en el mercado, en el esquema de producción centrada, y con costos elevados en la etapa de innovación, y en la rapidez con que se mueven y se mueren los productos (economía de la velocidad) (Srnicek, 2018). Por otro lado, centralmente en la producción de la fuerza de trabajo, se forma un polarización de la fuerza de trabajo (calificados/no calificados, exaltado por la lógica de la precariedad) y, solidarizándose con una subjetividad de gestión o managerial, introduce la dimensión de disrupción, de crítica, de aventura o de espontaneidad en la propia fuerza que se explota para la reproducción de capital (Zangaro, 2011).

Una "economía del comportamiento" (Sadin, 2020) se despliega en las empresas en la medida en que hay elementos de implicación subjetiva que, basado en datos, generan cursos de acción; partiendo del "giro conminatorio de la técnica" lo que se produce es una gestión de la vida según el algoritmo, que va en relación con los estándares de productividad (Sadin, 2020). Ahora, nuestra tesis es similar a la pistura de Szlechter y Zangaro (2020) que sostienen que estos elementos ya se encuentran en la década de 1930, con los estudios de Elton Mayo. En términos generales, el proceso desde los años '30 puede ser visto como una desmaterialización del trabajo y de las capacidades cognitivas y emocionales, lo que implica una desmaterialización del capital mismo. "El capital fijo pasó a constituir un saber almacenado, instantáneamente disponible por las TIC’s” (Szlechter \& Zangaro, 2020, p. 78). 
A su vez, podemos señalar que la "nueva forma de consumo productivo de la fuerza de trabajo supuso un giro subjetivo que dejaba de considerar el carácter como inmutable para pasar a concebir la subjetividad como un cúmulo de deseos, emociones y motivaciones a ser moduladas según las necesidades del capital” (Szlechter \& Zangaro, 2020, p. 78). Pese a la ascendencia de un discurso sobre la singularidad en el trabajo, o la demostración de lo que la persona es como condición para la adquisición de un puesto, es importante decir, para terminar, que las nuevas técnicas de dirección de empresa, y de producción de subjetividad, tienen como lógica el intercambio de datos, la construcción de curvas de variables de "cada uno" que se interrelacionan con "los demás". Se trata de un proceso de duplicación (a través de construcción de perfiles en las redes sociales, de test comandados por las people analitics e incluso en las nuevas disciplinas psi como lo son el coaching y la PNL donde el centro está puesto en la noción de conversación); es la duplicación del sujeto para su resingularización, resituarlo en una curva de enunciados capaz de hacer hablar y conducir al sujeto una vez establecida la vuelta. La nueva gestión de empresa encargada a inteligencias artificiales como las people analitics estudian las interacciones sociales, la posible relación de virtuales sujetos, y no comportamientos individuales. Si la puesta en práctica de los psicólogos en la década del '30 estaba centrada en las sombras del individuo, con potencial para gestionar la relación, la nueva empresa (reticular, algorítmica, sostenida por proyectos) puede atravesar con un lente al individuo, pero su objeto son las relaciones posibles entre ellos. Justamente es el data mining aquello que permite establecer esos "modelos analíticos relacionales" (Szlechter \& Zangaro, 2020, p. 79).

Por lo tanto, lo que está presente en el capitalismo informacional, como etapa de producción de bienes informacionales, es la asunción de los procesos de traducción mediante mecanismos digitales que posibilitan el pasaje de una emoción, un dato, a un cúmulo o curva de datos capaz de generar cursos de acción a través de sugerencias. Estas tecnologías producen información en el interior del despliegue del sujeto, en él mismo, "descubre" y traduce sus emociones y deseos, las relaciona con un cúmulo de variables afines, y vuelve como conducción de conducta. Se observa, pues, una íntima relación en las prescripciones del capitalismo emocional en las sociedades de la información en la medida en que los diversos espacios y procesos que registran y producen datos pueden ser utilizados para el "buen" intercambio de ciertas emociones en el contacto con algún otro abstracto y general que supone lo digital, con un sí mismo pasado o virtual, y con las mismas tecnologías digitales que reinsertan lo volcado en ellas mediante cursos de acción posibles que, por lo demás, se vuelven obligatorios.

Lo que se desprende de este apartado es la conmensurabilidad que adquiere las múltiples expresiones de la personalidad en cuanto a la información digital, es decir, la personalidad "coincide" y expresa por la variopinta producción de bienes informacionales primarios. Sin embargo, esto no es posible si la conectividad, la conexión y la atención captada de los otros no fuera un valor en sí mismo, o sea si no estuviera en el centro de la axiología del capitalismo. Suscita así la demostración, la publicitación de lo que uno "es" y, en ese sentido, los procesos de capitalización del cuerpo y de las emociones engloban a la subjetividad, se producen sujetos a escala global desde múltiples puntos de la red digital (para decirlo con Preciado). Ahora bien, sostenemos que la dividuación, la vuelta sobre sí mismo del sujeto y sobre otros, tiene como punto central para sostener la "ilusión de unidad" a la felicidad, el "mostrarse verdaderamente feliz" es un hecho que se repite en los múltiples ámbitos o red de relaciones donde se inserta el sujeto. A partir de esto, las nuevas técnicas de gestión de sí o tecnologías del yo perfilan 
herramientas y procedimientos para la unificación potencial de la multiplicidad de fragmentos por la cual el (in)dividuo se encuentra constituido.

\section{Consideraciones finales}

Pese a quedarnos algunas palabras en el tintero, algunos hilos detrás del archivo, hemos recorrido en estas líneas algunas elucubraciones teóricas para dar cuenta del capitalismo emocional en las grandes etapas discutidas actualmente, el industrial y el informacional. A la vez, hemos puesto en discusión las tecnologías del yo que se han sucedido, y que seguirán problematizándose en futuros trabajos. Igualmente, nos toca rescatar las conclusiones de lo que expuesto aquí.

En primer lugar, consideramos que la amplia producción dedicada a la gestión de las emociones, su direccionamiento, y su puesta en valor, deben ser abordadas desde una concepción que englobe el cuerpo y los cursos de acción. Así, hemos visto cómo las divisiones en el industrialismo, fundamentalmente operando como ortopedia social, direccionan al sujeto para su reconocimiento en la relación con un profesional, los cuadros medios de las fábricas. Estos logran hacerse cargo de la productividad por medio del dispositivo de la escucha; a la vez, supone la necesidad de examen y de confesión por parte de los trabajadores. Al colocar por fuera a las emociones, la narrativa psicológica permite la reformulación del sí mismo, del yo, por medio de una serie de operaciones de confesión-escucha. Esta habilidad será luego tenida en cuenta a la hora de seleccionar la fuerza de trabajo, fundamentalmente en la etapa informacional, no específicamente por lo que conlleva la producción de sus bienes, sino por el cambio en los modos de selección, la organización empresarial y por su axiología. A través de una serie de técnicas que permite la desingulazación o duplicación del trabajador, las empresas colocan en una curva de datos los posibles comportamientos: ya no es la confesión-escucha, sino que la tecnología permite recoger e intercambiar la información. Este es uno de los elementos del dividuo, directamente relacionado con los dispositivos de control: el fraccionamiento de sí mismo para su puesta en relación por medio de informaciones. El otro aspecto es la puesta en marcha de un sí mismo, una parte de sí, para conducirse: el impulso a ser mejor, la voluntad de la felicidad, las diversas sustancias que hay que controlar, son "subjetivizaciones" propias en post de la conducción de sí mismo, opera como tecnología del yo.

En términos generales, podemos decir que el ingreso al mercado laboral estará, desde la puesta en público del yo por parte de la narrativa terapéutica, plagada de requisitos emocionales, es decir, una puesta en marcha para la gestión de las emociones como cualidad necesaria para la asunción de un puesto de trabajo. Algunas corrientes de la sociología de las emociones y de los análisis críticos del capitalismo, plantean este requisito principalmente para la rama de servicios (atención al público, marketing, etc.), sin embargo, dada la evidencia de la popularización de la narrativa terapéutica y la consecuente ontología emocional que produce, se puede decir que la gestión de las emociones presenta una puesta en valor de la "personalidad" en cualquier trabajo. Más que depender del sector productivo, depende los instrumentos que se prescribe para la selección de la fuerza de trabajo: una entrevista con un psicólogo en un primer momento, luego en las tecnologías digitales que traducen expresiones, gestos, comentarios para realizar el perfil de la persona. Por otro lado, la cultura terapéutica ha introducido en la dinámica de productos culturales a las emociones en las mercancías. Es decir, la expansión de la narrativa terapéutica produjo una serie de mercancías emocionales que siempre se ven renovadas porque, en rigor, no 
hay un objetivo claro, un punto de llegada para "desistir" de consumir esos tipos de mercancías. Esto nos lleva a un tercer punto y consiste en la identificación de una falta, un problema o, en caso, una patología y la mercancía necesaria para sortear la falta (no llenarla); "sortear" puede ser utilizado como "manejar": se deben manejar las emociones, sino habrá alguna mercancía que nos permita hacerlo. Aquí tiene especial incidencia la farmacología y la psiquiatría biológica. La "cultura del Prozac", o de la aspirina en general, lleva a manejar dentro del umbral de lo óptimo, lo funcional y lo normal la vorágine emocional a la que se está sometido en la vida cotidiana, plagada de vicisitudes y cambios repentinos. El cuerpo como sistema de comunicación y particularmente el cerebro adquieren especificidad y dimensionalidad tal que exige mercancías que actúen en un marco delimitado; las sustancias devienen un elemento más para el manejo del yo y, por momentos, es esencial ${ }^{10}$.

Si la conmensurabilidad de las emociones establecidas por el capitalismo emocional, en la acepción industrial de los procesos productivos, iba expandiéndose a la par de los nuevos medios de comunicación o de la reubicación de las emociones en la literatura, en el capitalismo informacional, por su parte, permite una conmensuración "mayor" por el hecho de que las emociones pasarán a ser un bit más en ese mar de información digital. Además dado el influjo de las teorías biomédicas o farmacológicas las emociones pasaran a ser estados químicos, un equilibrio especifico de sustancias, y de forma general, de estímulos.

Mientras en el capitalismo industrial las técnicas de organización del yo producían sufrimiento y traumas que pretendían erradicar, en el capitalismo informacional, la literatura de autoayuda y la literatura management, focaliza en el individuo la capacidad de cambiar su situación que, también, dependió de él. La literatura psicológica permitía hacer un replanteamiento del yo para poder centrarse en los contextos (principalmente, la familia) donde esa persona devino como es, las nuevas discplinas psi, en cambio, pone el acento en la capacidad del propio sujeto de hacerse así mismo. Al desvincular al yo del contexto, sobrecarga al individuo o radicaliza la acepción de que puede cambiar lo que le sucede, en otras palabras, produce (otra) psicologización e individuación. A la par de esto, las exigencias sobre "creatividad", "pensamiento crítico", "emociones positivas" etc., exaltan la responsabilidad del trabajador y su dependencia emocional con la empresa. Los imperativos de transciéndete y muéstrate a ti mismo consisten en una dinámica que no tiene ningún punto de anclaje, donde la abstracción y la renovación son la marca por excelencia: no se sabe a quién se dirige ni qué repercute, por un lado, porque la velocidad vuelve obsoleta cualquier información-publicitación de sí, y, por otro, porque hay una serie de sugerencias invitando a la renovación y comparación consigo y con los demás por parte del algoritmo, entre otros factores. La lógica de la subjetividad como siempre dependiente de algo nuevo se introduce en un dispositivo que excita y frustra, está determinada por un ensamble que reactualiza incesantemente mecanismos de excitación-frustración ${ }^{11}$.

\footnotetext{
10 Parafraseando a Preciado, se puede preguntar qué viene primero la dopamina o la felicidad; en ese sentido hay dos reduccionismos que opera la narrativa terapéutica farmacológica, por un lado, la visión de lo positivo y lo negativo de las sustancias o de las cantidades de sustancias y, por otro, que dicha sustancia produzca un estado de ánimo, una totalidad más allá de la propia elaboración química.

${ }^{11}$ Un planteamiento similar puede seguirse en Dardot y Laval al referir al dispositivo de "rendimiento/goce", pero solo a condición de estar ligado a la dinámica de la producción, ya que es en la empresa donde se produce un continuo movimiento a rendir sumado de una experiencia de goce, incluso en un sentido lacaniano como un acercamiento a la muerte. El trabajador debe rendir más en cada proyecto de la empresa y a la vez dejar parte de sí, tender a la muerte, en el mismo proyecto (Dardot \& Laval 2013, p. 358). En otros términos, el trabajo se pornifica, adquiere una lógica de desecho del cuerpo (Preciado, 2008).
} 
Como un esbozo para trabajos posteriores, podemos señalar que es en la propia dinámica de la vida de los sujetos - una cotidianeidad plagada de mensajes motivacionales, sugerencias, registros, datos de sí mismo y diversas grillas de valorativas-, en esa vida cotidiana en cuya base se encuentra una lógica de excitación-frustración (estímulos "positivos" y resultados "negativos"), donde emergen diversos mecanismos de soportabilidad social. Esto va desde, por ejemplo, las narrativas terapéuticas, la ciencia de la felicidad, las sugerencias para el buen desarrollo de sí o el logro de objetivos otorgados por diferentes software (I am, Diamo, Pausa, etc.), o bien la reiterada publicación de sí para la validación de los demás. Ahora bien, los bienes informacionales primarios, aquellos que se suscitan por doquier en las redes digitales-móvilesvirtuales, expresan, condensan o "son" emociones y, sin duda, producen diversas formas de constituirse como sujeto, asumir una identidad y un cuerpo/emoción. Por eso mismo, el capitalismo emocional se encuentra exacerbado por lo informacional; desde el ángulo epistemológico, las emociones capaces de transformarse en mercancías o estímulos para el consumo encuentran su punto de contacto con lo informacional a través de la reducción de motivaciones, concepciones, situaciones determinadas en variables y, posteriormente, en un cúmulo de datos capaces de vender para publicidad, etc.; desde el lado práctico o, mejor dicho, pragmático, en cuanto desenvolvimiento en la vida cotidiana, los ámbitos y las relaciones pueden ser reducidas a ciertos quantum de elementos, desde los likes en redes sociales, las visitas o las interacciones hechas con los demás usuarios, hasta las tecnologías que otorgan preponderancia a las sustancias químicas y sus gradientes.

Entonces, la axiología del nuevo capitalismo, en la interrelación de la estructura organizacional flexible y competitiva, la producción centrada en los bienes informacionales y una tecnología que descompone en cantidades a los sujetos, está basada en el control de umbrales óptimos y funcionales, pero que no pueden ser estables sino que requiere de variaciones más o menos periódicas. Mientras que la estabilidad, la previsión, el ahorro, la carrera, el sacrificio y la proyección eran valores centrales en el capitalismo industrial, la asunción de riesgos, el cambio "planificado" o discrecional, el placer, la rapidez y la flexibilidad están a la orden del día en los mercados laborales y en las exigencias de la vida cotidiana actuales. Como la atención es efímera y escasa, la producción constante de sí como punto-de-atención, es decir, la producción de información digital sobre sí mismo tiene que ser constante y, sobre todo, "personal" a fin de atraer las miradas ajenas. La celebrity, mejor dicho, la influencers condensa esos valores, se muestra y transciende a sí misma al mismo tiempo que aúna puntos de observación y varía con intermitencia. En todo caso, cada axiología ha establecido sus límites y sus posibilidades, sus marcos de exclusión y de integración, y de lo que se trata, una vez más, es de la delgada línea de la agencia. Habrá que considerar en futuros trabajos las especificidades de estas nuevas coordenadas del yo. Lo que se hizo aquí es el marco teórico-simbólico de la asunción de las emociones por los capitalismos, y como toda operación por parte de esa maquinaria hay resistencias que se intentarán analizar posteriormente.

\section{Referencias}

Ahmed, S. (2019). La promesa de la felicidad. Katz.

Bauman, Z. (2009). Vida líquida. Paidós.

Bericat Alastuey, E. (2000) La sociología de la emoción y la emoción de la sociología. Papers 62, 145-176. 
Boltanski, L. y Chiapello, E. (2002). El nuevo espiritu del capitalismo. Akal.

Cabanas, E. (2013). La felicidad como imperativo moral. Origen y difusión del individualismo "positivo" en el capitalismo neoliberal y sus efectos en la construcción de la subjetividad. Tesis de Doctorado, Universidad Autónoma de Madrid. https:/ / repositorio.uam.es/handle/10486/662024

Cafassi, E. (1998). Bits, moléculas y mercancías. En Schiavo y Finquelievich (edits.), La ciudad y sus TICs: tecnologias de información y comunicación. Universidad Nacional de Quilmes.

Castel, R. (1997). La metamorfosis de la cuestión social. España.

Castells, M. (2006). La era de la información. La Sociedad Red. Siglo XXI.

Crary, J. (2015). 24 / 7: el capitalismo tardío y el fin del sueño. Paidós.

Dardot, P. y Laval, C. (2013). La Nueva Razón del Mundo: Ensayo sobre la sociedad neoliberal. Gedisa Editorial.

Deleuze, G. (1995). Post-scriptum sobre las sociedades de control. En Conversaciones 1972-199. Pre-textos.

Foucault, M. (1990). Tecnologías del yo y otros textos afines. Paidós.

Foucault, M. (2013). Para una política progresista no humanista. Respuesta a una pregunta. En ¿Qué es usted profesor Foucault? Sobre la arqueología y su método (193-222). Siglo XXI.

Foucault, M. (2004). Nacimiento de La Biopolítica: Curso en el Collège de France: 1978- 1979. FCE.

Foucault, M. (2006). Seguridad, territorio y población. FCE.

Gori, T. (2016). El valor en la época de su replicabilidad digital. Un abordaje a las teorías del capitalismo cognitivo y sus principales críticas. Hipertextos 4(6), 51-78.

Hochschild, A. (2008). La mercantilización de la vida intima: Apuntes de la casa y el trabajo. Katz.

Illouz, E. (2007). Intimidades congeladas: Las emociones en el capitalismo. Katz.

Illouz, E (2008). Saving the modern soul: Therapy, emotions, and the culture the self-help. University of California Press.

Illouz, E. (2009) Emotions, Imagination and Consumption. A new research agenda. Journal of Consumer Culture, 9 (3), 377-414.

Illouz, E. y Cabanas, E. (2019). Happycracia, Cómo la ciencia y la industria de la felicidad controlan nuestras vidas. Paidós.

Kogan, L. (2010). Hacia una teoría del cuerpo vivido y la identidad del yo. En Scribano, A. y Lisdero, P. (comp.) Las sensibilidades en juego: miradas múltiples desde los estudios sociales de los cuerpos $y$ las emociones. CEA-CONICET.

Marzano, M. (2011). Programados para triunfar. Nuevo capitalismo, gestión empresarial y vida privada. Tusquets.

MoulierBoutang, Y. (2011). Cognitivecapitalism. PolityPress.

Preciado, P. B. (2008). Testo Yonki. Espasa.

Remondino, G. (2012). Blog y redes sociales: un análisis desde las tecnologías de la gubernamentalidad y el género. Athenea Digital, 12 (3), 51-69

Rose, N. (2012). Políticas de la vida. UNIPE.

Rullani, E. (2004). El capitalismo cognitivo, ¿un dèjá-vu? En AA.VV Capitalismo cognitivo, propiedad intelectual y creación colectiva. Traficante de Sueños.

Sadin, E. (2020). La inteligencia artificial o el desafío del siglo. Anatomia de un antibumanismo radical. Caja Negra. 
Scribano, A. (2010). Primero hay que saber sufrir!!! Hacia una sociología de la espera. En Scribano, A. y Lisdero, P. (comp.) Las sensibilidades en juego: miradas múltiples desde los estudios sociales de los cuerpos y las emociones, CEA-CONICET.

Scribano, A. (2013). Sociología de los cuerpos/emociones. Revista Latinoamericana de Estudios sobre Cuerpos, Emociones y Sociedad, 10 (4), 93-113.

Sennett, R. (2000). La corrosión del carácter: Las consecuencias personales del trabajo en el nuevo capitalismo. Anagrama.

Sibilia, P. (2008). La intimidad como espectáculo. FCE.

Simon, H. A. (1971). Designing Organizations for an Information-Rich World. En Martin Greenberger (Edit.), Computers, Communication, and the Public Interest. Baltimore: The Johns Hopkins Press.

Srnicek, N. (2018). Capitalismo de plataformas. Caja Negra.

Szlechter, D. y Zangaro, M. (2020) Big data y people analytics: intimidad y emociones en la gestión de los recursos humanos. INNOV AR, 30 (78), 75-87.

Vercelone, C. (2013). Capitalismo cognitivo. Releer la economía del conocimiento desde el antagonismo capital-trabajo. Tesis 11. https:// halshs.archives-ouvertes.fr/halshs-00969302

Zangaro, M. (2015). Subjetividady trabajo. Una lectura foucaultiana del management. Herramienta.

Zukerfeld, M. (2010). Capitalismo y Conocimiento: Materialismo Cognitivo, Propiedad Intelectual y Capitalismo Informacional, Volumen I. Tesis doctoral. Equipo de Estudios sobre Tecnologías digitales, Capitalismo y Sociedad (E-TCS).

Zukerfeld, M. (2011a). El poder de la Atención. Materialidad y axiología de los flujos comunicativos en el Capitalismo Informacional. AVATARES de la comunicación y la cultura, (2).

Zukerfeld, M. (2011b). Obreros de los bits. Una introducción al sector información y el trabajo informacional. Editorial Jauretche.

Zukerfeld, M. (2017) Knowledge in the Age of Digital Capitalism: An Introduction to Cognitive Materialism. University of Westminster Press. 